\title{
DIAGNÓSTICO DE UN BANCO DE SANGRE, Y PROPUESTA DE MEJORA, HOSPITAL UNIVERSITARIO CLÍNICA SAN RAFAEL (HUCSR), BOGOTÁ - COLOMBIA
}

\author{
Ernesto Ciprian Guzmán \\ Escuela de Negocios, Gestión y Sostenibilidad \\ Programa de Especialización en Gestión Empresarial \\ Especialista en Gestión Empresarial \\ erciprian@poligran.edu.co \\ Laura Katherine Galeano Martínez \\ Escuela de Negocios, Gestión y Sostenibilidad \\ Programa de Especialización en Gestión Empresarial \\ Especialista en Gestión Empresarial \\ lkgaleano@poligran.edu.co \\ Mónica Marcela Bolívar \\ Escuela de Negocios, Gestión y Sostenibilidad \\ Programa de Especialización en Gestión Empresarial \\ Especialista en Gestión Empresarial \\ mmbolivar@poligran.edu.co
}

\section{Resumen}

El banco de sangre cumple una gestión importante dentro del sector salud, dado que tiene el rol de abastecimiento para los diferentes centros hospitalarios que requieran dicho insumo vital, el presente artículo analiza un caso de estudio particular de un hospital en la ciudad de Bogotá Hospital Universitario Clínica San Rafael (HUCSR). A partir de los análisis se identifican problemáticas que afectan el banco de sangre de la clínica, de esta forma se plantean diferentes propuestas de mejora para fortalecer su gestión. Metodología: la revisión parte del análisis de datos de la línea base de información sobre la recolección de sangre para el año 2017, incluyendo la experiencia de gestión operativa y administrativa de los funcionarios de la organización en la ejecución de esta actividad, posteriormente se realiza la búsqueda de los elementos teóricos necesarios para desarrollar el estudio; luego se efectúa un análisis de variables a través de Matriz DOFA. Finalmente, se proponen estrategias que buscan mejorar los procesos de comercialización de los hemoderivados producidos por el banco de sangre del (HUCSR), así como también plantear un plan de mejora para el robustecimiento y crecimiento de esta unidad de negocio dentro de la clínica. La importancia del presente estudio a nivel 
teórico radica en la aplicación de técnicas de ingeniería para mejorar procesos de la industria médica y de los servicios asociados a los hemoderivados.

Palabras clave: gestión de banco de sangre, unidad de negocio, Hemoderivado. 


\section{Introducción}

En el apartado de introducción se muestran los referentes teóricos de las temáticas principales para el desarrollo del presente caso de estudio.

\subsection{Teoría sobre gestión en bancos de sangre}

La sangre es el insumo principal para el funcionamiento de los bancos de sangre, ya que de ellos se obtienen diferentes hemoderivados "son medicamentos elaborados a partir del plasma humano, parte líquida de la sangre que se obtiene tras separar las células que contiene (glóbulos rojos, blancos y plaquetas)" (UNSAM, 2018) y otros componentes biológicos que se requieren para las terapias en la medicina transfusional, la cual se entiende como la "ciencia que tiene como objetivo la conservación y el restablecimiento de la salud apoyada en la terapéutica transfusional, una parte de la medicina que enseña el modo de tratar las enfermedades proporcionando los elementos sanguíneos celulares o plasmáticos que el enfermo requiera" (Narvaez, 2018). Sin embargo, para que ello se cumpla es necesario que exista una adecuada cadena transfusional, la cual se entiende como la selección adecuada de los donantes, la extracción de la sangre, el procedimiento y análisis de los componentes sanguíneos presentes y finalmente la transfusión para ser enviada al posible receptor o en su defecto a un banco de sangre.

Un Banco de Sangre "es todo establecimiento o dependencia con Licencia Sanitaria de Funcionamiento para adelantar actividades relacionadas con la obtención, procesamiento y almacenamiento de sangre humana destinada a la transfusión de la sangre total o en componentes separados, a procedimientos de aféresis y otros procedimientos preventivos, terapéuticos y de investigación. Tiene como uno de sus propósitos asegurar la calidad de la sangre y de sus derivados" (MinSalud, 2018), también puede definirse como una "organización técnico-científica y administrativa de una institución médica destinada a la transfusión de sangre y hemocomponentes provenientes de un banco de sangre" (INVIMA, 2018).

\subsection{Herramientas de aproximación a la problemática}

El análisis DOFA ha sido usado ampliamente para mejorar procesos en industria (Bernal N. M., 2018) y (Gutierrez, 2018), donde se toman como guía herramientas como (Ramírez, 2018) que detallan los pasos a tener en cuenta al momento de realizar un análisis de este tipo.

Una herramienta que nos permite conocer el estado actual del banco de sangre es el diagnóstico empresarial, dado que tiene como objetivo evaluar, controlar y mejorar todas aquellas actividades relacionadas con el negocio (Noda, 2009). Tener una fuente base de trabajo permite establecer los parámetros y los mecanismos que se tendrán en cuenta para abordar el problema.

Se parte de la realización de un diagnóstico, el punto de partida o línea base y se generan propuestas que encaminen al cambio que se requiere, alineando estas con la plataforma estratégica de la organización. El propósito básico de la etapa de diagnóstico es llegar a una clara comprensión de la situación actual y la identificación de las problemáticas o pocos vitales; considerando la recolección de datos y el análisis de los mismos; adicionalmente el planteamiento de mejoras de las actividades y su relación de dependencia con varios factores, como la naturaleza del problema o las necesidades, tanto internas como externas (Arenas, 2018). 
Por lo tanto, un diagnóstico empresarial le permite a la alta dirección tener una radiografía detallada del estado actual de la organización para así maximizar las fortalezas hasta el punto de reducir las debilidades, con el fin de impulsar una estrategia de ventaja competitiva provocando el aprovechamiento de las oportunidades y la reducción las amenazas; todo ello con el fin de generar la mejora en la gestión de la organización (Bernal D. D., 2014) . Visto de otra manera, es este diagnóstico el que facilita la toma de decisiones frente a estrategias futuras, siendo este un instrumento guía para evaluar el éxito o fracaso del programa o proyecto (Ricaurte, 2018).

Un instrumento bastante útil para este tipo de ejercicios es un "Análisis DOFA", dado que es una herramienta que se utiliza para reconocer las amenazas del entorno y así generar las acciones tanto preventivas como correctivas, ver la reacción de la organización frente a estas y otras posibles situaciones que pueden presentarse. Identificar las fortalezas de la organización posibilita su aprovechamiento, reconocer nuevas oportunidades de negocio, mitigar las debilidades de la compañía, evaluar la rentabilidad de las distintas decisiones, entre otras (Bernal N. M., 2018). Este facilita que se abarquen los aspectos más relevantes como lo son los factores del entorno donde se mueve la organización con el fin de observar su comportamiento frente al mercado (amenazas - oportunidades) y de igual manera revisar los factores internos que permiten evaluar aspectos como clima organizacional, imagen institucional, objetivos, procedimientos, tecnología, productos, etc., (fortalezas - debilidades) que se encuentran bajo el control de la organización.

El fin último de dicho análisis es plantear estrategias y acciones que sean para su beneficio, sin embargo, éstas deben surgir de un proceso de estudio detallado y un encadenamiento de recursos, igualmente estas deben ser explícitas para alcanzar los objetivos propuestos; deben considerar también las diferentes alternativas que se tendrán en cuenta para dar solución al problema dado, que involucra también diferentes fuentes de apoyo para la ejecución de este, como también los efectos que estos generarán, los costos presentes y futuros, como los riesgos que ello implicará, la resistencia (interna y externa) que se pueda presentar, etc. En definitiva, esto es lo vital de este ejercicio: que las estrategias propuestas se alineen con la competitividad de la organización, dado que es esta la que caracteriza a una compañía de otra, porque ese valor añadido es el que hace que sea tenida en cuenta por sus clientes, distinguiéndola en el mercado y frente a sus potenciales competidores (Ramírez, 2018).

La forma más adecuada de implementar dichas estrategias será a través de un Plan de Mejoras el cual constituye aquellas decisiones estratégicas que se deben reunir e incorporar a cada uno de los procesos de la compañía para que éstos sean percibidos por el cliente como un mejor servicio. El plan es la base de trabajo para la detección de mejoras y el planteamiento tanto de acciones correctivas como preventivas y minimizar el impacto de los riesgos no previstos (ANECA, 2018). Generar un cambio en cualquier organización demanda un esfuerzo conjunto entre la dirección estratégica y el talento humano el cual permitirá el cumplimiento de objetivos y metas. El plan de mejora como proceso se usa para adquirir la calidad total y la excelencia de las empresas de manera paulatina, para así alcanzar resultados eficientes y eficaces. Su objetivo principal radica en obtener una estrecha relación entre los procesos organizacionales y el talento humano generando una sinergia que contribuya de manera positiva en un progreso constante (Proaño, 2017).

\section{Caso de estudio}


El Banco de Sangre del Hospital Universitario Clínica San Rafael (HUCSR) (Imagen 1) de la ciudad de Bogotá, es uno de las instituciones con reconocimiento a nivel nacional, debido a que es una entidad de 4 to nivel que ofrece servicios en diferentes especialidades de alta calidad.

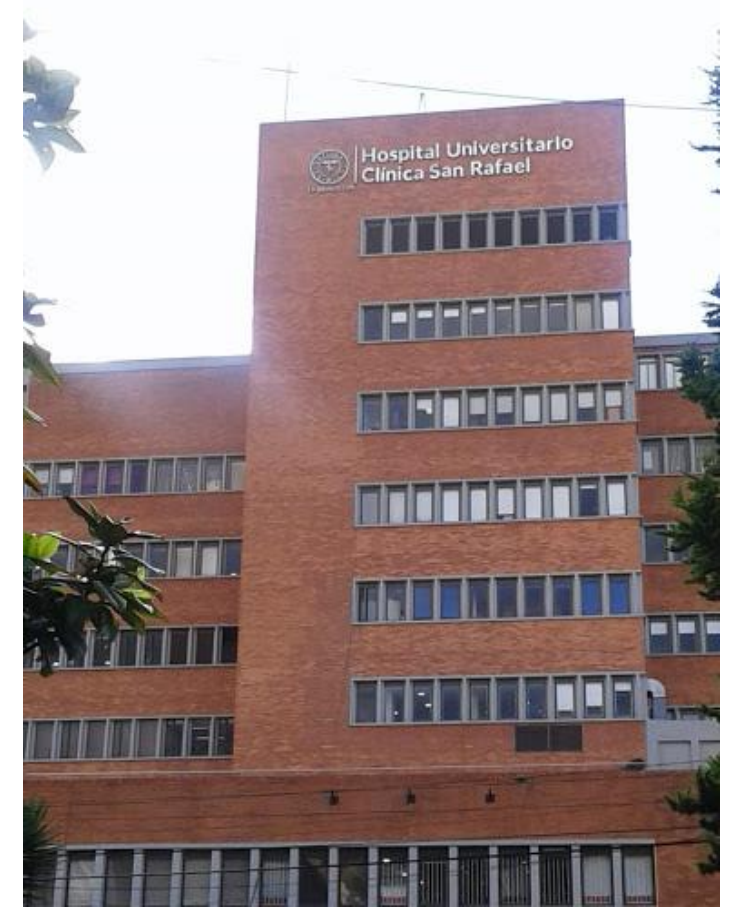

Imagen 1: Fotografía (HUCSR) Bogotá, 2018

Fuente Propia.

El Banco de Sangre del (HUCSR) fue diseñado inicialmente como un área de apoyo diagnóstico y terapéutico que prestaba sus servicios únicamente a los pacientes que ingresaban al (HUCSR), es decir eran beneficiarios directos de todos los procesos y procedimientos del banco, lo que genero para la clínica un reconocimiento por su calidad en servicio e insumos directos. Sin embargo, con el pasar del tiempo y al ver que se estaba generando una gran numero de hemocomponentes, se presentó la oportunidad y el Banco de Sangre posterior a un estudio del mercado y adquisición de clientes, se transformó en un centro de distribución de hemoderivados para diferentes entidades de salud en el país, que requieren de dichos insumos para su funcionamiento.

Por lo tanto, generó crecimiento económico en gran medida por la venta de componentes y la competitividad que provocó el banco de sangre objeto de estudio; fue afortunada pues se posicionó con gran liderazgo y esfuerzo frente a los demás bancos de sangre de la ciudad, como el 4to banco (Véase Tabla 1) más importante de distribución de hemoderivados para Bogotá - Colombia. 


\begin{tabular}{|l|}
\hline PUESTO ENTIDAD \\
\hline 1 Sociedad Nacional de la Cruz Roja \\
\hline 2 Instituto nacional de Cancerologia \\
\hline 3 Hospital Militar \\
\hline 4 Hospital Universitario Clinica San Rafael \\
\hline 5 Hospital Universitario de la Samaritana \\
\hline 6 Sociedad de Cirugia de Bogota - Hospital de San Jose \\
\hline 7 Fundacion Cardio Infantil \\
\hline 8 Fundacion Hospital de la Misericordia \\
\hline 9 Clinica Marly \\
\hline 10 Clinica Colsanita \\
\hline 11 Hospital Central Policia Nacional \\
\hline 12 Hemocentro Distrital \\
\hline 13 Fundacion Hematologica de Colombia \\
\hline 14 Fundacion Karl Landsteiner \\
\hline 15 Hemolife \\
\hline
\end{tabular}

Tabla 1: Posicionamiento Bancos de Sangre Bogotá 2017

Fuente. Elaboración Propia

Haciendo una revisión del Banco de Sangre del (HUCSR), de sus procesos y las estrategias corporativas, se detecta que la capacidad actual del banco en cuanto a infraestructura, maquinaria, personal y captación de sangre se están quedando cortas frente a la posibilidad de crecimiento que hay en el mercado de hemocomponentes, y reconocimiento como organización tanto en el distrito capital como en el país. Actualmente el banco de sangre tiene un promedio de facturación favorable (Gráfico 1), generando así expectativas para el crecimiento.

Para controlar la actividad de los bancos de sangre “...El sistema nacional de transfusiones debería estar regido por políticas y marcos legislativos nacionales relativos a la transfusión de sangre para promover la uniformidad de las normas y de la cualidad y la seguridad de la sangre y los productos derivados de la sangre". (OMS, 2018). En Colombia se han establecido algunas normativas para este ejercicio profesional como se ve en la (imagen 2), en esta imagen se presentan aquellas que rigen el servicio de los bancos de sangre en Colombia. 


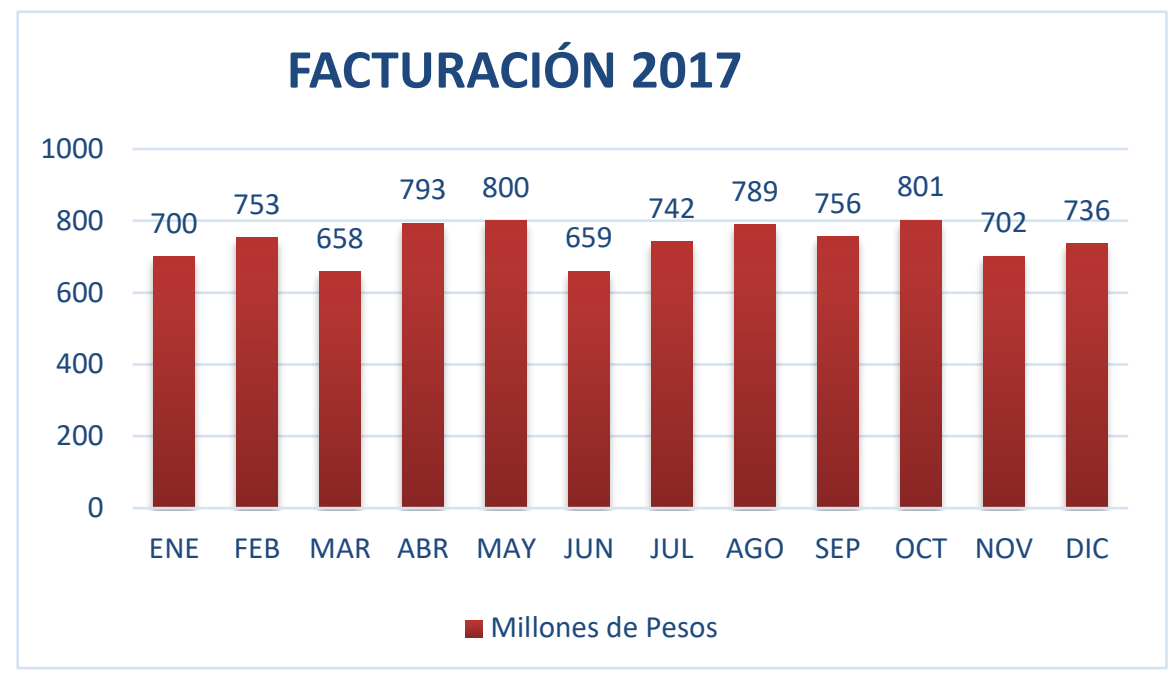

Gráfico 1: Facturación 2017 (HUCSR),

Fuente. Elaboración Propia
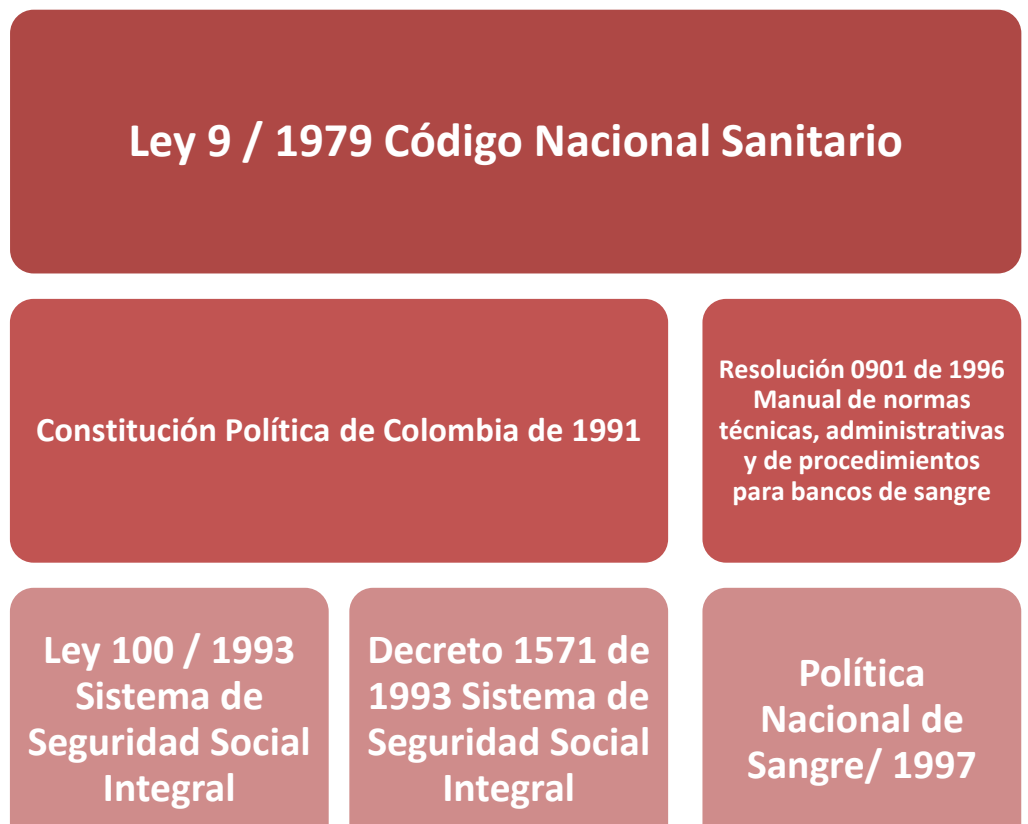

Decreto 1571 de 1993 Sistema de Seguridad Social Integral

Política

Nacional de Sangre/ 1997

Imagen 2: Normatividad para la Cadena Transfusional

Fuente. Elaboración Propia

\section{Metodología}

En este apartado se describe paso a paso la forma en que se enfrentó el caso de estudio con sus problemáticas identificadas, y el aporte en relación a las soluciones brindadas. En la (imagen 3) se 
muestra la metodología con la que se ejecutó el estudio del banco de sangre del (HUCSR) abarcando todos los aspectos relevantes que ello implica.

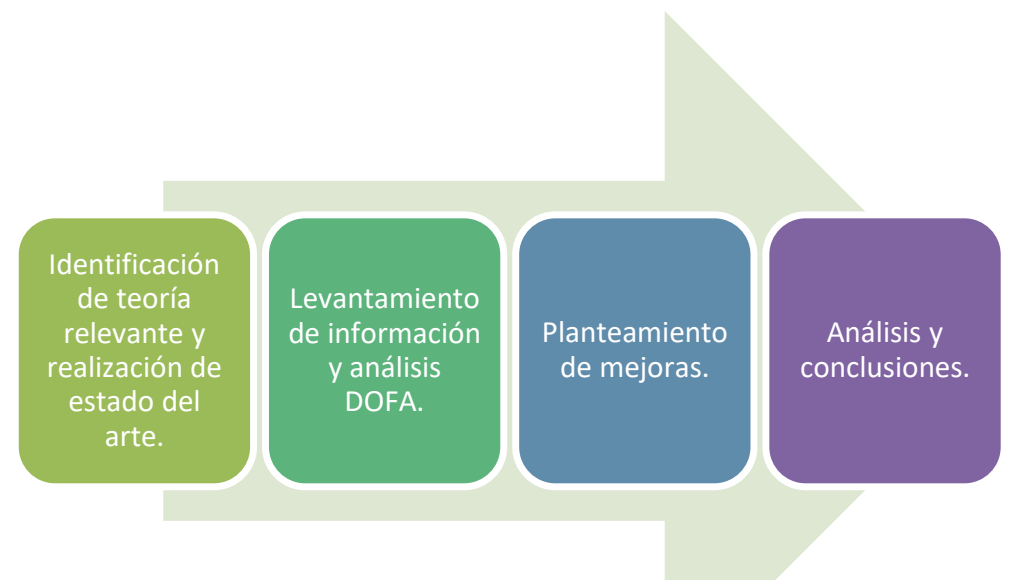

Imagen 3: Metodología ejecución de estudio, Fuente. Elaboración Propia

\subsection{Levantamiento de Información}

Para entender de manera adecuada el procedimiento del banco de sangre y poder generar el diagnóstico correspondiente, se realizó una entrevista a la Coordinadora del Banco de Sangre del (HUCSR) Dra. Leila Marisol Barrera (Barrera, 2018), en donde nos explica paso a paso el proceso tanto operativo como administrativo, adicionalmente se obtienen datos estadísticos donde se identifican cifras representativas que permiten crear una estrategia para el cubrimiento de la demanda. Dentro de los aspectos relevantes se destacan los equipos de alta tecnología y el avanzado sistema de información que permite ofrecer un servicio de calidad, seguro, oportuno y eficiente frente al análisis de las muestras de los procesos efectuados.

\subsection{Caracterización de Procesos del Banco de Sangre}

A continuación, se detalla cómo se realiza el proceso del banco de sangre en el (HUCSR)

\subsubsection{Captación de Sangre}

El Banco de Sangre del (HUCSR) realiza captación de sangre (imagen 4) por medio de campañas extramurales, es decir, ubican carpas en puntos estratégicos para recolectar la mayor cantidad de sangre, de lunes a viernes, ubican 4 carpas, el sábado 2 carpas y el domingo 1 carpa (imagen 5).

Los puestos de recolección están dotados tanto de equipos como de talento humano capacitado, auxiliares de enfermería, jefes de enfermería, bacteriólogos y médicos generales, de acuerdo con la normatividad (MinSalud, 2018). 


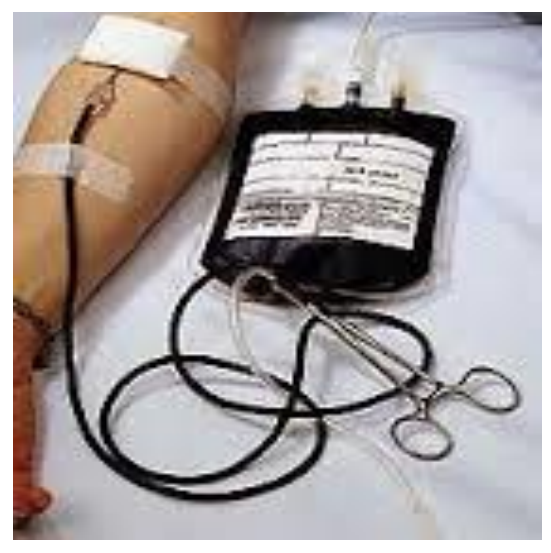

Imagen 4: Fotografía (HUCSR) Fuente. Propia

Bogotá, 2018

Para dar inicio al proceso de captación y garantizar el procedimiento, se realiza una encuesta al donante que se divide en: encabezado, datos personales, factores de riesgo y consentimiento. Adicionalmente el banco para control de cada donante tiene dos formatos que corresponden a la ficha clínica y la autoexclusión con lo cual se garantiza un procedimiento de calidad, tranquilidad y satisfacción tanto para el donante como para el banco de sangre.

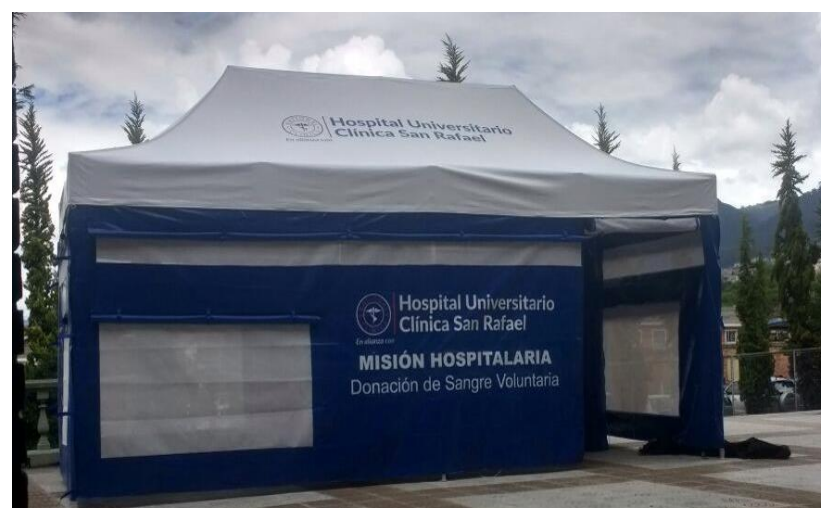

Imagen 5: Fotografía (HUCSR) Fuente Propia, Bogotá, 2018

Se clasifican algunos tipos de donante con el fin de hacer una valoración previa del riesgo de presentar una Reacción adversa a la Donación (Saludcapital, 2018):

Autólogo. Persona que dona su sangre completa o uno de sus componentes, con el fin de ser transfundida posteriormente. Requiere consentimiento informado.

Dador por Reposición: persona que se acerca a un banco de sangre a dar su sangre total o sus hemocomponentes, con el fin de reponer sangre que se va a transfundir o se transfundió a un paciente conocido, compañero de trabajo, vecino o familiar.

Dirigido Específico o Intrafamiliar: dona su sangre para un receptor determinado, por exigencia del paciente, de sus familiares o del médico tratante. 
Autoexcluido pos-donación: una vez ha realizado donación de sangre total o hemocomponentes, comunica al BS, de manera inmediata o posterior a la donación que su sangre no sea utilizada con fines transfusionales.

Los diferentes donantes asisten a diversas campañas para la recolección de sangre, esto permite un buen funcionamiento del banco de sangre en relación al aprovisionamiento, las habilidades y estrategias de posicionamiento de las carpas que son centros móviles para donación, permiten generar en promedio de 2000 a 3000 unidades de sangre (Gráfico 2).

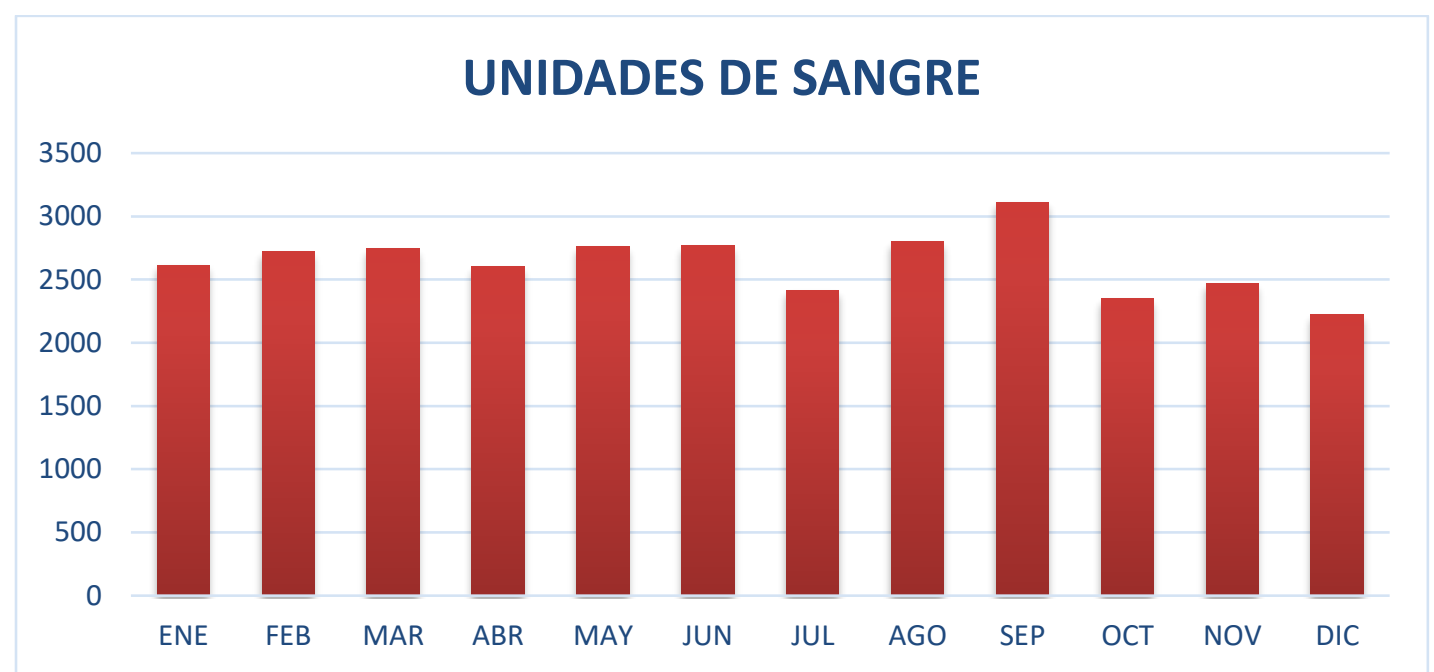

Gráfico 2: Unidades de Sangre recolectadas por mes 2017 (HUCSR)

Fuente. Elaboración Propia

\subsubsection{Preparación de Compontes}

Una vez analizadas las unidades de sangre, se realiza el proceso de fraccionamiento (Imagen 6), el cual consiste en tomar cada unidad de sangre recolectada y extraer los componentes esenciales para la venta.

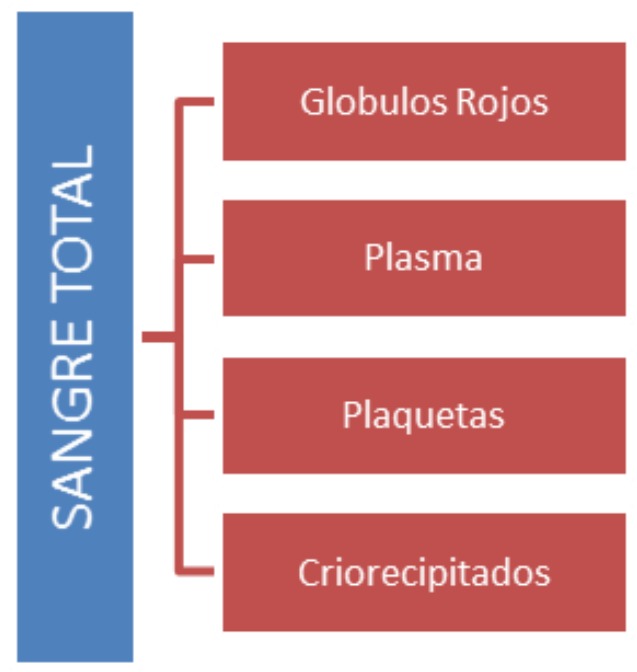

Imagen 6: Hemoderivados, Fuente. Elaboración Propia 
Estos elementos son los que ingresan al mercado, se ofertan a entidades que requieren realizar la transfusión de cualquiera de los componentes, en cualquier parte del país, generando aproximadamente el fraccionamiento de 7000 componentes (Gráfico 3) los cuales se extraen con equipos especializados que funcionan 24 horas al día, realizando el fraccionamiento correspondiente y listo para el respectivo proceso de conservación y almacenamiento.

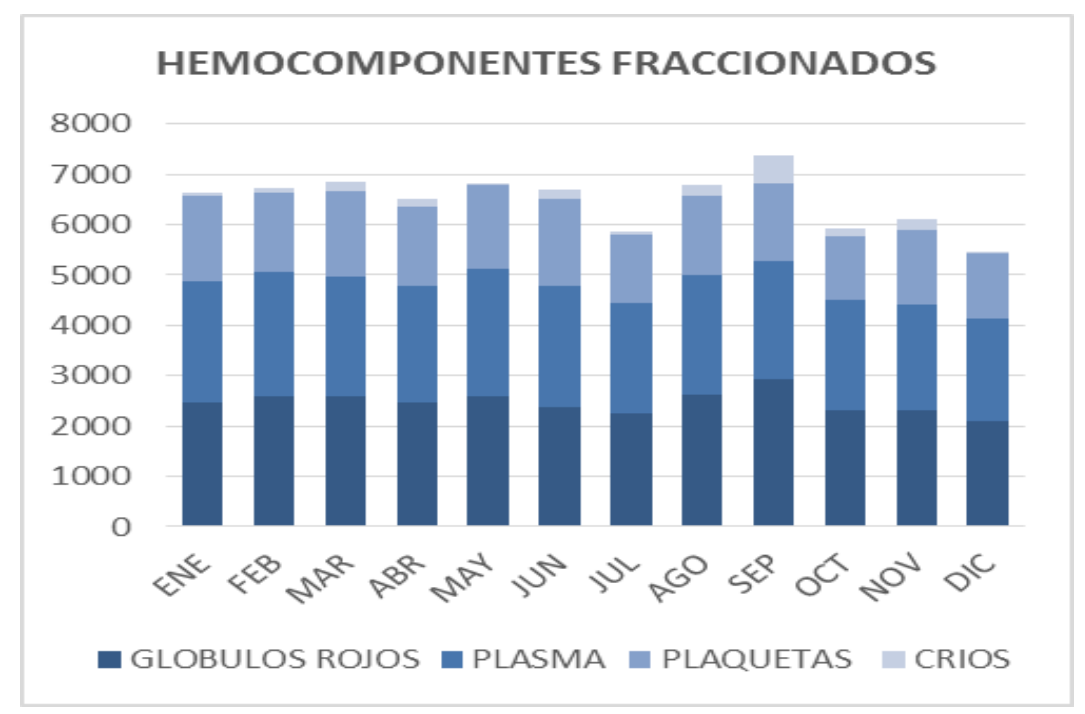

Gráfico 3: Fraccionamiento de Hemoderivados por mes 2017 (HUCSR), Fuente. Elaboración Propia

Una vez realizado este proceso de fraccionamiento se realizan muestras para validar cualquier reactivo negativo en la sangre, que impida la transfusión de componentes a los receptores, estas pruebas corresponde a:

$\checkmark$ Prueba serológica para sífilis.

$\checkmark$ Detección de anticuerpos contra el virus de la hepatitis C.

$\checkmark$ Detección de anticuerpos contra el antígeno Core de la hepatitis B (MinSalud, 2018)

$\checkmark$ Detección del antígeno de superficie del virus de la hepatitis B.

$\checkmark$ Serología para Tripanosoma cruzi.

$\checkmark$ Detección de anticuerpos contra el virus de la Inmunodeficiencia Humana Adquirida (HIV) 1 y 2.

$\checkmark$ Detección de anticuerpos contra el virus de HTLV I/II (MinSalud, 2018) Prueba

Las cuales son establecidas por ley para dar seguridad al paciente que recibirá el componente a la hora de la transfusión, en general la reactividad es mínima (Gráfico 4). Sin embargo, es importante teniendo en cuenta que es el control de calidad que se hace a las unidades, adicional a los estándares de conservación. 


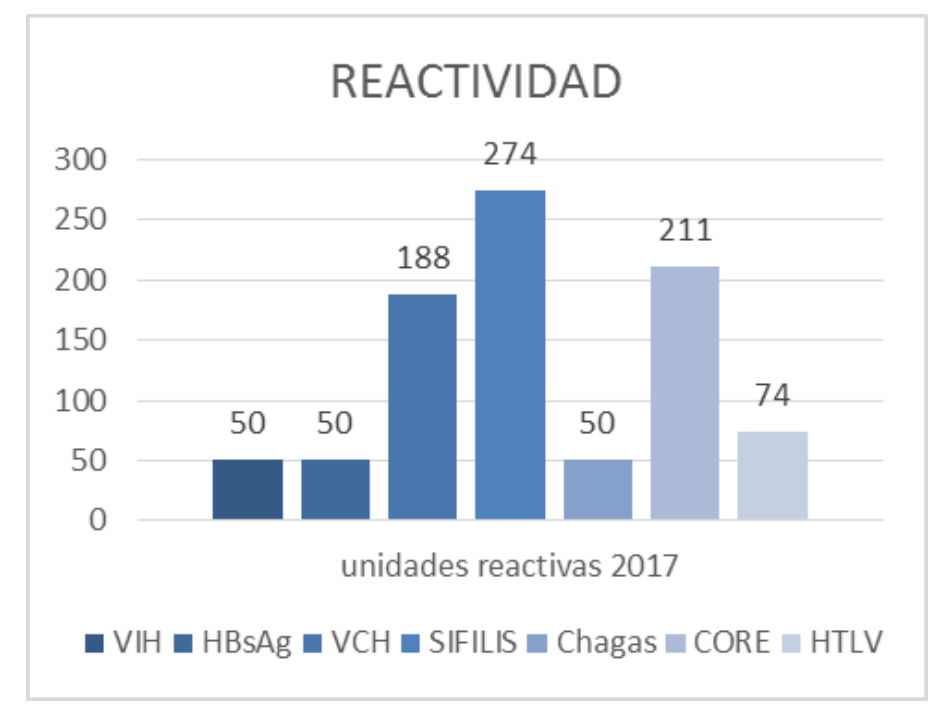

Gráfico 4: Unidades reactivas 2017 (HUCSR), Fuente. Elaboración Propia 3.2.3 Transfusión

Se realiza el procedimiento al paciente, de acuerdo a los componentes que requiere, con el fin de suplir el faltante en el cuerpo, combatiendo enfermedades como anemia, hepatitis, leucemia, entre otras. Para dar cumplimiento al proceso de transfusión se debe tener en cuenta la normatividad vigente imagen 7 (MinSalud, 2018), con el fin de prestar un excelente servicio.

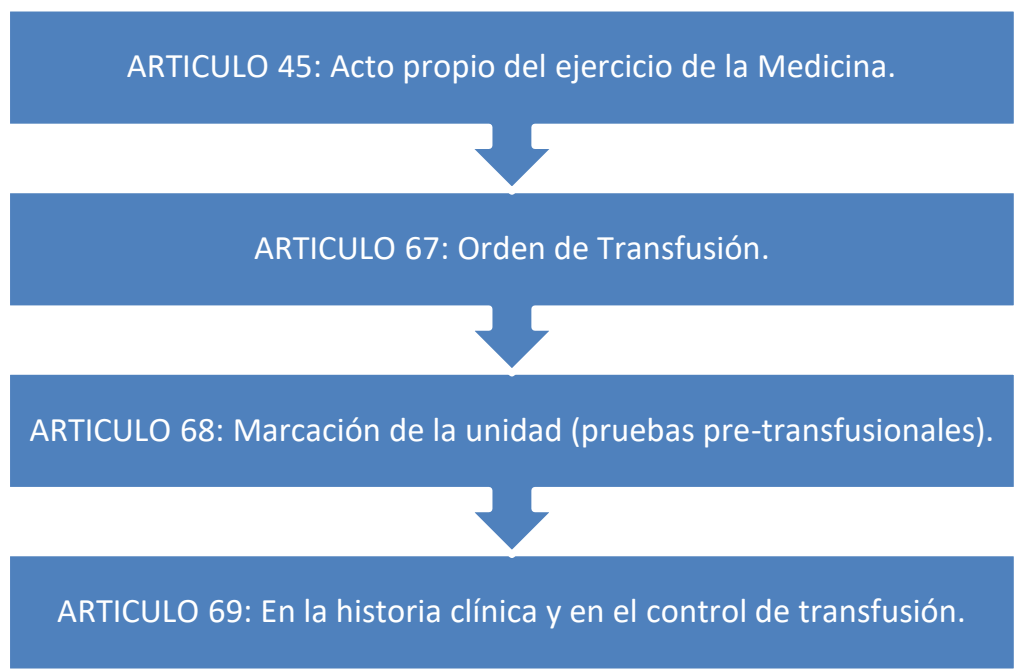

Imagen 7: Decreto 1571 DE 1993, Fuente. Elaboración Propia

Al momento de realizar el procedimiento, puede que el paciente no acepte dicha transfusión, es decir que el cuerpo no reconozca el componente y se generen reacciones adversas al procedimiento, en este caso se debe realizar el siguiente esquema (imagen 8): 


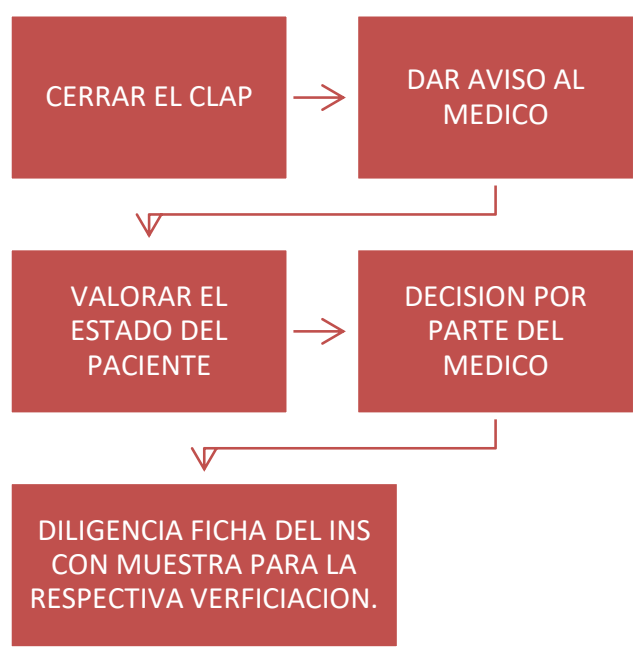

Imagen 8: Esquema de procedimiento reacciones adversas en la transfusión, Fuente. Elaboración Propia

Si no se presenta ningún evento adverso, termina el proceso de transfusión y se considera exitoso.

\subsection{Distribución de Hemoderivados}

El Banco de Sangre del (HUCSR) tiene 26 contratos transfusionales con diferentes entidades en todo el país dentro de los que se destacan Clínica Nogales, Hospital Militar, Clínica Federmann, IPS Esimed Tunja (Clínica Tunja) entre otros, con esta actividad el Banco se ha posicionado como uno de los bancos más grandes, durante los últimos años se han distribuido alrededor de 40.000 componentes (Gráfico 5) a nivel nacional, generando así una rentabilidad tanto para el (HUCSR) como para el propio Banco de Sangre.

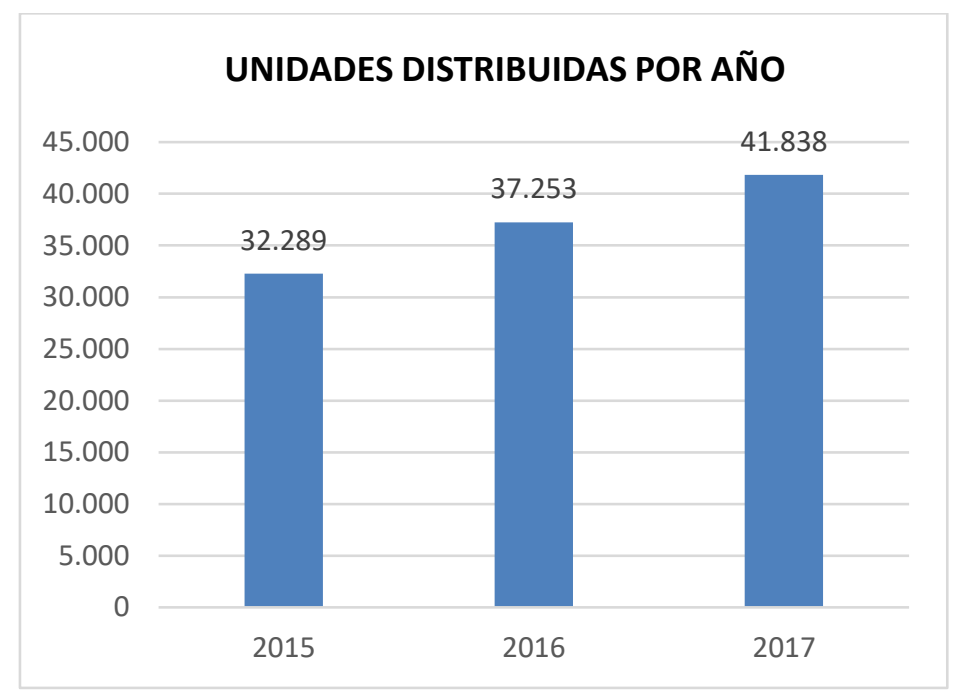

Gráfico 5: Unidades Distribuidas 2017 (HUCSR), Fuente. Elaboración Propia 


\subsection{Análisis del Entorno del banco de sangre (HUCSR)}

El análisis del entorno (imagen 9) se enfocó principalmente en dos esferas: macro entorno y micro entorno ya que esta actividad se ve influenciada por diferentes sectores.

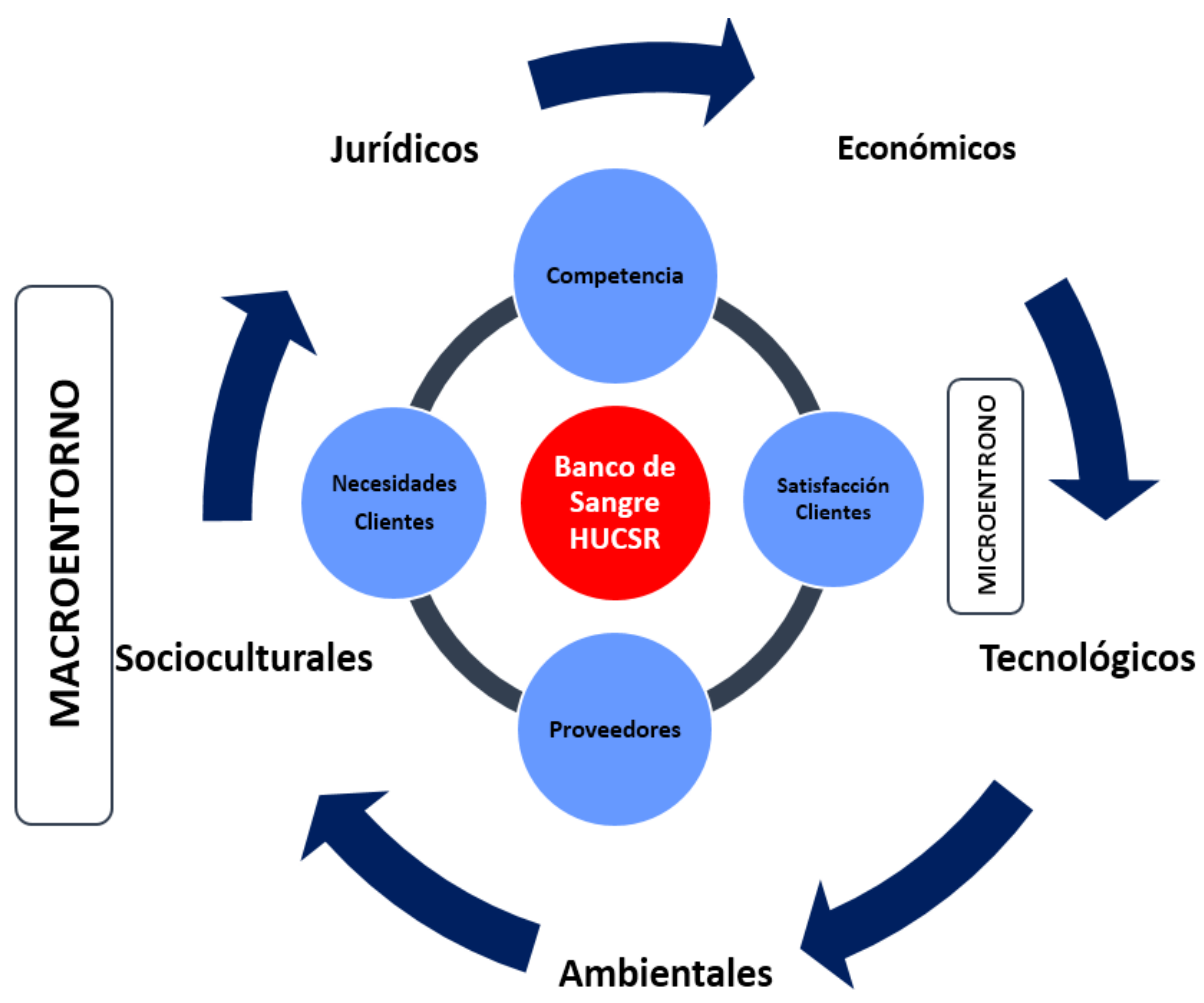

Imagen 9: Análisis del entorno (HUCSR), Elaboración Propia

Se establece de esta manera toda vez que para el desarrollo de esta actividad influyen cantidad de factores, como por ejemplo, la demanda, la estructura, las tendencias, la actualización de conocimientos, las estrategias, los eventos adversos de la naturaleza, etc.

El banco de sangre del (HUCSR) puede generar una ventaja competitiva a nivel local, generando la prestación de servicios con un concepto diferencial, en cuanto a calidad, precio y cantidad de unidades recolectadas. 


\subsection{Matriz DOFA}

Tabla 2: Matriz DOFA (HUCSR)

\section{OPORTUNIDADES}

- Investigar el mercado para poder adquirir contratos para la venta de componentes.

- Se realizan diferentes actividades para llamar la atención de los donantes en los meses críticos.

- Se entrega calidad de los componentes sanguíneos extraídos para los clientes.

- Departamento de calidad el cual apoya y verifica todos los procesos organizacionales, administrativos y de gestión de la organización.

- Adquirir contratos para poder aumentar la venta de los hemoderivados.

DEBILIDADES

- No contar con una infraestructura suficiente para la debida atención de la demanda.

- Deficiencia de donantes frente a la proyección de crecimiento esperado.

- Perdida de oportunidad de reconocimiento en el mercado.

- Demora en la habilitación y adecuación del nuevo punto de servicio.

- La sangre recolectada no cumpla con los requisitos para la extracción de los componentes.

- Afectación en la recolección de sangre en los periodos de vacaciones (Junio - Diciembre).

- No contar con una gestión de mercadeo para la divulgación de venta de hemocomponentes y la imagen corporativa del banco.

\section{- FORTALEZAS}

- Certificación de calidad que le permite encontrarse en el 4 lugar en el ranking nacional, siendo el único hospital que vende componentes.

- El banco desarrolla campañas extramurales para la variedad en la captación de donantes.

- Destreza en la trasformación de componentes sanguíneos, para su proceso de fraccionamiento.

- Ubicación estratégica para la ejecución del proceso y la distribución de productos.

- Se cuenta con implementos necesarios y suficientes para ejecutar el debido proceso.

- Profesionales calificados y en permanente actualización que los hace competitivos frente al mercado.

\section{- AMENAZAS}

- No se de una planeación financiera para el crecimiento en un proyecto a largo plazo.

- Mayor crecimiento de los bancos de sangre existentes.

- Mitos existentes de la donación de sangre, haciendo que las personas se abstengan de realizar el proceso.

- El proceso de la captación de sangre solo sede para un grupo sanguíneo.

- Entes de control impidan la apertura de nuevos bancos de sangre.

- Se pueda presentar la no renovación de la certificación de salubridad por parte del INVIMA

- Abastecimiento de sangre en eventos catastróficos.

Fuente. Elaboración Propia 
Dados los hallazgos obtenidos en la matriz DOFA se destacan los siguientes: i) se evidencia que la mayor oportunidad que presenta el banco de sangre es la de continuar con la adquisición nuevos contratos para la venta de componentes sanguíneos, lo que se ve como un crecimiento continuo; obligando indirectamente al banco a tener una muy buena base de materia prima para cumplir con la demanda requerida, ii) de ahí que se considere como debilidad latente la infraestructura actual, tanto física como tecnológica, ya que impide que se cumplan los requerimientos de la potencial demanda, y que hace indispensable considerar una amenaza importante que impida el adecuado desarrollo de dicho proyecto, iii) al no tener definida una adecuada planeación financiera para el largo plazo.

\section{Plan de Mejora}

El propósito de este estudio es analizar la posibilidad de crecimiento del banco de sangre del (HUCSR), teniendo en cuenta los aspectos vistos en la matriz DOFA, la cual nos permite visualizar con más detalle las ventajas y las desventajas que el plan pueda presentar.

\subsection{Acciones}

Lo podemos definir como los conceptos a tener en cuenta para el plan de mejora, y que son importantes para su implementación:

a) Recursos Financieros.

b) Cantidad de sangre necesaria para cumplir a los clientes.

c) Capacidad tecnológica y de talento humano, que se encuentren en mejora continua.

Una vez plateados estos aspectos y una vez aprobado por el (HUCSR) y con los recursos disponibles para iniciar el trabajo, el plan de mejora consiste en ampliar la sede con la que actualmente se cuenta, conseguir otro equipo que permita el fraccionamiento de más hemoderivados, contratar el mejor talento humano que permita garantizar la calidad, para lograrlo necesitamos:

$\checkmark$ Compra o arrendamiento de una instalación más amplia con capacidad para la tecnología (imagen 10) que se requiere en el proceso de fraccionamiento de hemocomponentes, el cual se desea incrementar en un 50\% del procesamiento actual, es decir recolectar aproximadamente 4.500 unidades por mes y fraccionar 10.500 hemocomponentes.

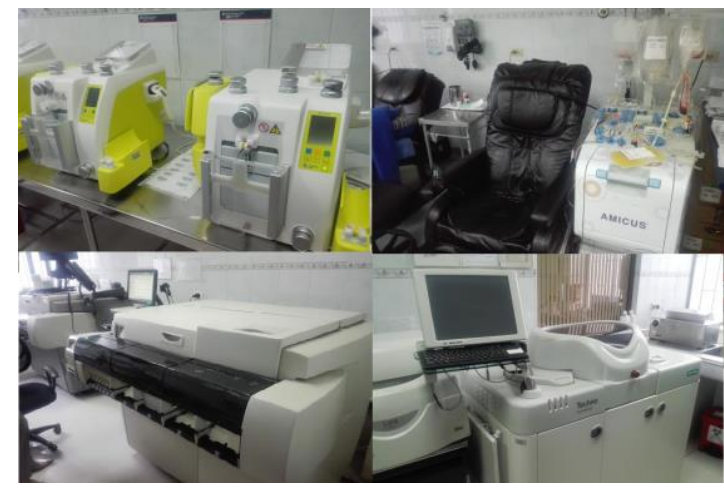

Imagen 10: Equipos procesamiento Banco de Sangre (HUCSR), Fuente Propia 
$\checkmark$ Generar un fortalecimiento de imagen institucional, que permita la obtención de nuevos clientes que potencialicen la actividad del banco de sangre

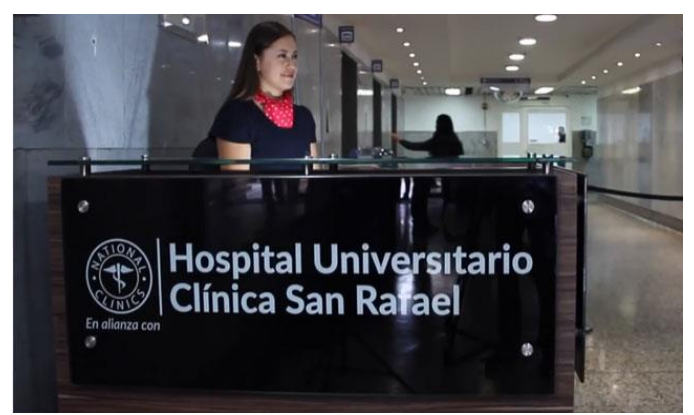

Imagen 11: Fuente. (HUCSR, 2018)

Actualizar el sistema de información, con el fin de llevar el control de cada unidad y hemocomponentes, permitiendo evidenciar de forma estadística el crecimiento.

$\checkmark$ Fortalecer las campañas extramurales para mayor captación de unidades de sangre.

\subsection{Impacto}

El banco brindara crecimiento tanto en sus instalaciones como en el proceso que ejecutara, lo cual mostrara el aumento de captación de donantes y por ende unidades para fraccionar, lo que lo hace llamativo frente al mercado ya que podrá obtener más contratos de venta de los componentes sanguíneos y a su vez puede escalar en el ranking.

A nivel social se generará empleo debido a que se contratará personal adicional para que ejecute el proceso, en el cual se adecuaran más equipos y se tendrá materia prima adicional de la que se obtiene hasta el momento, lo cual favorece al sector del mercado laboral y ayudara al crecimiento que tendrá el banco.

Se dará mejor calidad de vida a aquellas personas que requiere de transfusiones, ya sea por una desafortunada enfermedad que presente o cualquier otro evento adverso que pueda afectar la salud, por lo cual es conveniente incentivar la donación voluntaria desvirtuando los mitos presentes en la comunidad generando una mayor conciencia de los beneficios que genera esta actividad.

\section{Conclusiones}

Esta investigación logra mostrar todo lo que rodea el buen funcionamiento del banco de sangre, la importancia de una buena administración, que permiten el apoyo diagnóstico oportuno y el suficiente abastecimiento de hemocomponentes a entidades externas, sin que se limite únicamente al mal concepto de receptor de unidades de sangre y no genere otros procesos.

Es importante reconocer, que el insumo central para el crecimiento del Banco de Sangre del (HUCSR) son las donaciones de sangre y las campañas que se realizan para recolectar cada unidad, hay que tener en cuenta que esta actividad también se ve afectada, pues existen varias razones que generan complicaciones para la captación de la base central de trabajo, algunas de estas son mitos, creencias religiosas e incluso hábitos que hacen que muchas de las personas que son aptas para donar, no lo realicen. 
Aunque la captación de sangre es importante, sin embargo una gran dificultad para el crecimiento del banco es no contar con una independencia financiera, toda vez que es un banco dependiente del (HUCSR), y al no contar con los recursos propios, no puede generar un crecimiento autónomo; también puede ser un obstáculo, el no tener una fuerza de mercadeo permanente que permita promover el reconocimiento externo del Banco de Sangre.

El banco de sangre del Hospital Universitario Clínica San Rafael, presenta una gran ventaja y es que a pesar de su dependencia y de sus instalaciones pequeñas, tiene la cobertura de más de 26 IPS en el país, por lo cual es catalogada como uno de los bancos más grandes de distribución a nivel distrital, esto gracias a la captación de sangre y al personal especializado que realiza cada proceso.

La investigación de cara al objetivo primordial, es generar el crecimiento del banco de sangre ubicándose en un espacio con mayor capacidad e infraestructura tecnológica, con el fin de llegar a más clientes, manteniéndonos en el mercado con reconocimiento.

La meta es que esté en funcionamiento a inicios del año 2021, aplicando adecuadamente el plan de mejora propuesto el cual después de varios análisis dan visión al proyecto. 


\section{Bibliografía}

ANECA, A. N. (16 de 06 de 2018). Universidad de Antofagasta. Obtenido de http://www.uantof.cl/public/docs/universidad/direccion_docente/15_elaboracion_plan_de_mej oras.pdf

Arenas, F. (16 de 06 de 2018). Universidad de Chile, Ingeniería Industrial. Obtenido de http://www.dii.uchile.cl/ eolguin/G\%20de1\%20C/trabajos/Fernando\%20Arenas.doc.

Barrera, L. M. (05 de 06 de 2018). Gestión Administrativa y Operativa Banco de Sangre (HUSCR). (G. L. Bolivar Mónica, Entrevistador)

Bernal, D. D. (2014). La alternativa del diagnóstico empresarial para la gestión directiva en las pequeñas empresas comerciales en Sinaloa. TELOS. Revista de Estudios Interdisciplinarios en Ciencias Sociales, 6.

Bernal, N. M. (16 de 06 de 2018). Fundación Universitaria Konrad Lorenz. Obtenido de http://www.konradlorenz.edu.co/images/publicaciones/suma_negocios_working_papers/2012v2-n1/02_pegantes_urano.pdf

Gutierrez, J. C. (23 de 06 de 2018). Biblioteca Digital, Universidad EAN. Obtenido de http://repository.ean.edu.co/bitstream/handle/10882/1703/VegaAlex2012.pdf?sequence=3

HUCSR, H. U. (23 de 06 de 2018). Obtenido de http://www.clinicasanrafael.com/

INVIMA, A. B. $(10$ de 06 de 2018). INVIMA. Obtenido de https://www.invima.gov.co/images/stories/ABCpublicidad/ABC-Bancos-de-Sangre.pdf

Jiménez, M. R. (10 de 06 de 2018). Banco de Sangre Laboratorio. Obtenido de https://libroslaboratorio.files.wordpress.com/2011/10/banco-de-sangre.pdf

MinSalud, $\quad$ M. $\quad$ d. $(10$ de 06 de 2018). Obtenido de https://www.minsalud.gov.co/Normatividad_Nuevo/DECRETO\%20\%201571\%20DE\%201993 .pdf

MinSalud, M. $\quad$ d. $\quad$ (16 de $06 \quad$ de 2018$)$ Obtenido de https://www.minsalud.gov.co/sites/rid/Lists/BibliotecaDigital/RIDE/DE/DIJ/RESOLUCION_0 901_de_1996.pdf

MinSalud, M. d. (16 de 06 de 2018). Recuperado el 16 de 06 de 2018, de www.minsalud.gov.co/Normatividad_Nuevo/Resoluci\%C3\%B3n\%200437\%20de\%202014.pdf

MinSalud, M. $\quad$ d. $(23$ de 06 de 2018). Obtenido de https://www.minsalud.gov.co/Normatividad_Nuevo/DECRETO\%20\%201571\%20DE\%201993 .pdf

Narvaez, M. J. (10 de 06 de 2018). Repositorio Institucional UNAM-Managua. Obtenido de http://repositorio.unan.edu.ni/1014/1/57886.pdf

Noda, G. A. (2009). Propuesta de una herramienta diagnóstico para evaluar la compresión de la Gestión de la Información y el Conocimiento en la empresa en perfeccionamiento Diseño Ciudad Habana. Ciencias de la Información, 14.

OMS, O. M. (16 de 06 de 2018). Disponibilidad y seguridad de la sangre a nivel mundial. Obtenido de http://www.who.int/es/news-room/fact-sheets/detail/blood-safety-and-availability

Proaño, D. X. (2017). METODOLOGÍA PARA ELABORAR UN PLAN DE MEJORA CONTINUA. 3 C Empresa, 50 - 56.

$\left.\begin{array}{lllllll}\text { Rafael, } & \text { H. } & \text { U. } & \text { (10 } & \text { de } & 06 & \text { de }\end{array}\right)$. http://www.clinicasanrafael.com.co/contenido.asp?tabla=servicios_y_especialidades2.

Ramirez, J. L. (17 de 06 de 2018). UNIVERSIDAD VERACRUZANA. Obtenido de ROJAS, JOSÉ LUIS RAMIREZ: https://www.uv.mx/iiesca/files/2012/12/herramienta2009-2.pdf 
Ramírez, J. L. (16 de 06 de 2018). Universidad Veracruzana, Instituto de Investigaciones y Estudios Superiores de las Ciencias Administrativas. Obtenido de https://www.uv.mx/iiesca/files/2012/12/herramienta2009-2.pdf

Ricaurte, C. Q. (16 de 06 de 2018). Universidad Nacional de Río Negro. Obtenido de http://unrn.edu.ar/blogs/pt/files/2013/03/6-Ricaurte09-GuiaPlanTuris-ver-fichas-al-final-post2007.pdf

$\begin{array}{llllllll}\text { Saludcapital, } & \text { S. } & \text { S. } & (16 \quad \text { de } & 06 & \text { de } & \text { 2018). Obtenido de }\end{array}$ http://www.saludcapital.gov.co/DDS/Documentos\%20Red\%20Sangre/PROTOCOLO\%20RA D\%20Y\%20GUIA\%20DE\%20MANEJO.pdf

UNSAM, U. N. (10 de 06 de 2018). Obtenido de http://www.unsam.edu.ar/tss/con-la-innovacion-en-lasangre/ 